\title{
A Novel Analytic Technique for the Service Station Reliability in a Discrete-Time Repairable Queue
}

\author{
Renbin Liu ${ }^{1}$ and Yinghui Tang ${ }^{2}$ \\ ${ }^{1}$ School of Mathematics and Statistics, Chongqing University of Technology, Chongqing 400054, China \\ ${ }^{2}$ School of Mathematics and Software Science, Sichuan Normal University, Chengdu 610066, China \\ Correspondence should be addressed to Renbin Liu; liurb888@126.com and Yinghui Tang; tangyh@uestc.edu.cn
}

Received 13 February 2013; Accepted 11 May 2013

Academic Editor: Zhijun Liu

Copyright (c) 2013 R. Liu and Y. Tang. This is an open access article distributed under the Creative Commons Attribution License, which permits unrestricted use, distribution, and reproduction in any medium, provided the original work is properly cited.

\begin{abstract}
This paper presents a decomposition technique for the service station reliability in a discrete-time repairable Geom ${ }^{X} / \mathrm{G} / 1$ queueing system, in which the server takes exhaustive service and multiple adaptive delayed vacation discipline. Using such a novel analytic technique, some important reliability indices and reliability relation equations of the service station are derived. Furthermore, the structures of the service station indices are also found. Finally, special cases and numerical examples validate the derived results and show that our analytic technique is applicable to reliability analysis of some complex discrete-time repairable bulk arrival queueing systems.
\end{abstract}

\section{Introduction}

Queues with service station subject to failures and repairs, so-called repairable queues, are often encountered in many practical applications such as in computer, manufacturing systems, and communication networks. Because system performance deteriorates seriously by service station failures and the limitation of repair capacity, the study of queues with service station failures and repairs is not only important for theoretic investigations, but it is also necessary for engineering applications.

Relative to continuous-time repairable queues, their discrete-time counterparts received very little attention in the literature. Moreover, most of discrete-time repairable queueing models were investigated from a queueing theory viewpoint only, and some quantities relative to queueing theory, such as queue size, waiting time, and busy period, were obtained. But the reliability quantities of the service station, such as the unavailability and failure frequency of the service station, were neglected (see, e.g., [1-11]).

On the other hand, to the best of our knowledge, the existing work about the discrete-time repairable queues was studied with the help of the discrete supplementary variable technique, the matrix-geometric solution method, and the decomposition method. The discrete supplementary variable technique has important applications in queueing theory. With this method, various repairable queueing systems were investigated in the existing literatures (see, e.g., $[1-8]$ ). The matrix-geometric solution method studied by $\mathrm{Yu}$ [9] and other researchers is a more powerful tool. For example, Yu et al. [10] applied this method to analyze the characteristics of the $\mathrm{MAP} / \mathrm{PH}(\mathrm{PH} / \mathrm{PH}) / 1$ queue with repairable server where lifetime of server, service time, and repair time of server are all discrete phase-type random variables. The decomposition technique first introduced by Tang et al. [11] is a novel probabilistic method, and it is based on renewal process theory and $z$ transforms and is different from the above two methods. Tang et al. [11] use this method to analyze the queue length distribution and its stochastic decomposition property for a discrete-time $\mathrm{Geo}^{X} / \mathrm{G} / 1$ repairable queueing system with multiple adaptive delayed vacations. Recently, they have also obtained several reliability quantities of the service station [12].

The purpose of this paper is to introduce Tang's decomposition technique for the reliability of a discrete-time repairable Geom ${ }^{X} / \mathrm{G} / 1$ queueing system, in which the server 

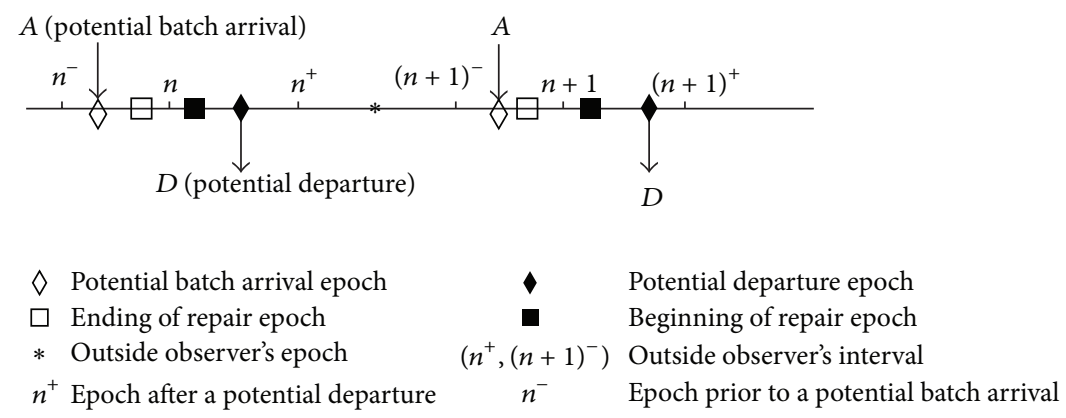

FIGURE 1: Various time epochs in a late arrival system with delayed access.

takes exhaustive service and multiple adaptive delayed vacation discipline, and the service station is subject to failures and repairs. According to such a novel analytic technique, we discuss the main reliability indices of the service station, including the probability that the time $n$ is during server busy period, the unavailability and the average failure number during $(0, n]$. What is more, we derive some important reliability relation equations, which indicate the structures of the service station indices. It should be noted that these reliability relation equations are new results and are not obtained by the existing discrete analytic techniques, such as the discrete supplementary variable technique [1-8] or matrix-geometric solution method $[9,10]$. Some special cases and numerical examples validate the derived results and show that Tang's decomposition technique is applicable to reliability analysis of some complex discrete-time repairable bulk arrival queues.

The rest of the paper is organized as follows. The next section gives the model assumptions and some preliminaries. In Section 3, we use Tang's decomposition technique to study the main reliability indices of the service station, including the probability that the time $n$ is during server busy period, the unavailability and the average failure number during $(0, n]$. We also derive some important reliability relation equations, which indicate the structures of the service station indices. Sections 4 and 5 present some special cases and numerical examples. Conclusions are finally drawn in Section 6.

\section{Assumptions and Preliminaries}

We consider the following discrete-time Geom ${ }^{X} / G / 1$ queueing system with multiple adaptive delayed vacations and repairable service station.

(1) Let the time axis be slotted into intervals of equal length with the length of a slot being unity. To be more specific, let the time axis be marked by $0,1, \ldots, n, \ldots$. Here, we discuss the model for a late arrival system with delayed access (LAS-DA), and therefore a potential batch arrival takes place in $\left(n^{-}, n\right)$, and a potential departure occurs in $\left(n, n^{+}\right)$; for details, see Hunter [13]. The various time epochs involved in our model can be viewed through a self-explanatory figure (see Figure 1). The interarrival times between bulk customers, $\left\{\tau_{i}, i \geq 1\right\}$, are independent identically distributed (i.i.d) random variables generated by a geometric distribution $P\left\{\tau_{i}=j\right\}=p(1-p)^{j-1},(j \geq 1,0<p<1)$. Each group size $D$ is a random variable satisfying distribution $P\{D=k\}=e_{k}, k \geq 1$ with finite mean $e$ and probability-generating function (PGF) $A(z)=\sum_{k=1}^{\infty} e_{k} z^{k}$, $|z|<1$.

(2) The customers in different bulk arrivals are served in FCFS order, and the order in one bulk arrival is arbitrary. At a time, the server only serves a customer at the service station. The service times, $\left\{\chi_{n}, n \geq 1\right\}$, are i.i.d random variables having distribution $g_{j}=P\left\{\chi_{n}=j\right\}, j \geq 1$ with PGF $G(z)=\sum_{j=1}^{\infty} g_{j} z^{j},|z|<1$ and average service time $\mu$.

(3) The server will take at most $H$ vacations when the system becomes empty. Before each vacation, the server will spend some time for preparation. If a batch of customers arrives in the preparation (delayed) time, the server will abort the upcoming vacation and begin to serve the customers immediately. A vacation will be taken if no customer arrives in delayed time. Upon returning from a vacation, the server immediately serves if there is a waiting queue at the service station or prepares for another vacation if there is an empty queue at the service station, and the number of vacations taken is less than $H$. If the total $H$ vacations have been finished and no arrival occurs, the server will stay idle and wait for the customer arrival.

(4) Assume that $H$ obeys an arbitrary distribution $P\{H=$ $j\}=h_{j}, j \geq 1$ with PGF $H(z)=\sum_{j=1}^{\infty} h_{j} z^{j},|z|<1$. Denote the server's the $i$ th vacation time by $V_{i}, i \geq 1$. Suppose that $V_{i}, i \geq 1$ are independent and follow an identical arbitrary distribution $v_{j}=P\left\{V_{i}=j\right\}, j \geq 1$ with PGF $v(z)=\sum_{j=1}^{\infty} v_{j} z^{j}$, $|z|<1$. The delayed times, denoted by $\left\{W_{i}, i \geq 1\right\}$, are i.i.d discrete random variables having an arbitrary distribution $w_{j}=P\left\{W_{i}=j\right\}, j \geq 1$ with PGF $w(z)=\sum_{j=1}^{\infty} w_{j} z^{j},|z|<1$.

(5) The service station may possibly fail when and only when it is serving a customer. The failed service station will be repaired by the server immediately. After repair, the service station is as good as new and continues to serve the customer whose service has not been finished 
yet. We assume that the service time for a customer is cumulative.

(6) The lifetime $X$ of the service station has a geometric distribution $P\{X=j\}=\alpha(1-\alpha)^{j-1},(j \geq 1,0<\alpha<$ $1)$, and its repair time $Y$ obeys an arbitrary distribution $y_{j}=P\{Y=j\}, j \geq 1$ with PGF $y(z)=\sum_{j=1}^{\infty} y_{j} z^{j},|z|<1$ and a mean repair time $\beta$.

(7) All random variables are mutually independent. At the initial time $n=0$, the server begins to serve when the number of customers presented in the system $N(0)>0$, or the server is idle and waits for the first arrival when $N(0)=0$.

Remark 1. From the assumptions (3) and (7), we know that after the first busy period, the server will begin to prepare for a vacation. Obviously, the assumption (7) is practical and reasonable. However, with Tang's decomposition technique, we will later prove that the steady-state reliability indices of the service station are independent of the initial state $N(0)=$ $i, i \geq 0$, and they have nothing to do with multiple adaptive delayed vacations.

Remark 2. The multiple adaptive delayed vacation presented by this paper is introduced by Tang et al. [11], which is a generalization of multiple adaptive vacations [14]. The analysis in this work shows that Tang's method is applicable to reliability analysis of some complex discrete-time repairable queueing systems with generalized vacation.

Throughout this paper, we adopt the following notations. $E(X)$ is the mean of random variable $X . P(Q)$ is the probability of event $Q . C_{l}^{k}=l ! /(k !(l-k) !), 0 \leq k \leq l$.

Definition 3. The "generalized service time of a customer" $\tilde{\chi}$ denotes the time interval from the time when the server begins to serve a customer until the service of this customer ends, which includes some possible repair times of the service station due to its failures in the process of serving this customer.

Lemma 4 (see [11]). The PGF and the mean of $\tilde{\chi}$ are given, respectively, by

$$
\begin{gathered}
\widetilde{G}(z)=\sum_{j=0}^{\infty} P(\tilde{\chi}=j) z^{j}=G[z \alpha y(z)+z(1-\alpha)], \quad|z|<1, \\
E(\tilde{\chi})=\left.\frac{d \widetilde{G}(z)}{d z}\right|_{z=1}=\mu(1+\alpha \beta),
\end{gathered}
$$

where $G(z)=\sum_{j=1}^{\infty} g_{j} z^{j}$ and $y(z)=\sum_{j=1}^{\infty} y_{j} z^{j}$ are given by assumptions (2) and (6).

Definition 5. The "server busy period" denotes the time interval from the time when the server begins to serve a customer until the system becomes empty, which contains some possible repair times of service station due to its failures in the process of service.

Let $\widetilde{b}$ denote the server busy period initiated with one customer and let its PGF be $\tilde{b}(z)=\sum_{j=0}^{\infty} P(\tilde{b}=j) z^{j},|z|<1$. According to [15], the following lemma holds.

Lemma 6. $\widetilde{b}(z)$ satisfies the equation $\widetilde{b}(z)=\widetilde{G}[(1-p) z+$ $p A(\widetilde{b}(z)) z]$, and

$$
E(\widetilde{b})= \begin{cases}\frac{\mu(1+\alpha \beta)}{[1-p e \mu(1+\alpha \beta)]}, & \tilde{\rho}<1, \\ \infty, & \tilde{\rho} \geq 1,\end{cases}
$$

where $\tilde{\rho}=p e \mu(1+\alpha \beta)$ is the traffic intensity of the considered queueing system.

The server busy period initiated with $i$ customers is denoted by $\tilde{b}^{\langle i\rangle}$. Then, $\tilde{b}^{\langle i\rangle}$ can be expressed as $\tilde{b}^{\langle i\rangle}=\tilde{b}_{1}+\cdots+\tilde{b}_{i}$, where $\widetilde{b}_{1}, \ldots, \widetilde{b}_{i}$ are independent of each other and have the same distribution as $\tilde{b}$. Thus, the PGF of $\widetilde{b}^{\langle i\rangle}$ is $\widetilde{b}^{i}(z)$.

Definition 7. The "idle period of the system" denotes the time interval from the time the system becomes empty until bulk customers arrive and enter the system.

Let $I_{k}$ denote the $k$ th idle period of the system, and then according to the system assumptions, $\left\{I_{k}, k \geq 1\right\}$ are mutually independent and follow an identical geometric distribution $P\left(I_{k}=j\right)=p(1-p)^{j-1}, k, j=1,2,3, \ldots$

Lemma 8 (see [13]). If $|z|<1, c_{n}>0$ and $c(z)=\sum_{n=0}^{\infty} c_{n} z^{n}$, then

$$
\lim _{z \uparrow 1}(1-z) c(z)=c<\infty \Longleftrightarrow \lim _{n \rightarrow \infty} \frac{1}{n} \sum_{k=1}^{n} c_{k}=c<\infty .
$$

Lemma 9 (see [13]). If $\lim _{n \rightarrow \infty} c_{n}=c<\infty$, then $\lim _{n \rightarrow \infty}(1 / n) \sum_{k=1}^{n} c_{k}=c$.

\section{Reliability Analysis of the Service Station}

In this section, with the help of Tang's decomposition technique, we will discuss main reliability indices of the service station and analyze their structures.

\subsection{The Probability That the Time $n$ Is during Server Busy Period}

Theorem 10. Let $A_{i}(n)=P$ (the time $n$ is during server busy period $\mid N(0)=i), i \geq 0$, and let $a_{i}(z)=\sum_{n=0}^{\infty} A_{i}(n) z^{n},|z|<$ 1 , and then for $i \geq 0$, the PGF of $A_{i}(n)$ is 


$$
\begin{aligned}
a_{0}(z)=\frac{\tau(z)}{1-z}\{1- & \frac{A(\widetilde{b}(z))[1-w(\bar{p} z) v(z)-w(\bar{p} z) H(\theta(z))(v(\bar{p} z)-v(z))]}{1-t(z)} \\
& \left.+\frac{A(\widetilde{b}(z)) \tau(z)[1-w(\bar{p} z)+H(\theta(z))(w(\bar{p} z)-\theta(z))]}{1-t(z)}\right\}, \\
a_{i}(z)=\frac{1}{1-z}\left\{1-\frac{\widetilde{b}^{i}(z)[1-w(\bar{p} z) v(z)-w(\bar{p} z) H(\theta(z))(v(\bar{p} z)-v(z))]}{1-t(z)}\right. & \\
& \left.+\frac{\tilde{b}^{i}(z) \tau(z)[1-w(\bar{p} z)+H(\theta(z))(w(\bar{p} z)-\theta(z))]}{1-t(z)}\right\}, \quad i \geq 1 .
\end{aligned}
$$

Further, one has

$$
\lim _{n \rightarrow \infty} A_{i}(n)=\lim _{z \uparrow 1}(1-z) a_{i}(z)= \begin{cases}\operatorname{pe\mu }(1+\alpha \beta), & \tilde{\rho}<1, \\ 1, & \tilde{\rho} \geq 1,\end{cases}
$$

where

$$
\begin{gathered}
\bar{p}=1-p, \quad \tau(z)=\frac{p z}{1-\bar{p} z}, \quad \theta(z)=w(\bar{p} z) v(\bar{p} z) \\
\Lambda(z)=\bar{p} z+p z A(\widetilde{b}(z)), \\
t(z)=A(\widetilde{b}(z)) \tau(z)[1-w(\bar{p} z)+H(\theta(z))(w(\bar{p} z)-\theta(z))] \\
+w(\bar{p} z) v(\Lambda(z))+w(\bar{p} z) H(\theta(z)) \\
\times(v(\bar{p} z)-v(\Lambda(z))) .
\end{gathered}
$$

And $w(z), v(z), A(z), H(z), \widetilde{b}(z)$, and $\widetilde{\rho}$ are given by assumptions (1)-(6), Definition 5, and Lemma 6, respectively.

Proof. Let

$$
\begin{gathered}
s_{k}=\sum_{i=1}^{k}\left(W_{i}+V_{i}\right), \quad \delta_{k}=\sum_{i=1}^{k} \tau_{i}, \quad k \geq 1, \quad s_{0}=\delta_{0}=0, \\
\sum_{m[i]=i}^{\infty}=\sum_{m_{1}=1}^{\infty} \cdots \sum_{m_{i}=1}^{\infty}, \quad m[i]=\sum_{j=1}^{i} m_{j},
\end{gathered}
$$

and $\xi(n)=1$ represents the event that the time $n$ is during server busy period. Denote by $I_{i}$ the $i$ th idle period of the system with a geometric distribution (see Definition 7), and $\tilde{b}^{\langle k\rangle}$ is the server busy period initiated with $k$ customers with PGF $\widetilde{b}^{k}(z)$ (see Lemma 6). Since the ending points of server busy period and server vacation are renewal points, by noting that the server stays idle and waits for the first arrival when $N(0)=0$, we get

$$
A_{0}(n)=P\left(I_{1} \leq n, \xi(n)=1\right)
$$

$=\sum_{k=1}^{\infty} e_{k} P\left(I_{1} \leq n<I_{1}+\widetilde{b}^{\langle k\rangle}\right)$

$+\sum_{k=1}^{n} e_{k} \sum_{j=1}^{\infty} h_{j} P\left(I_{1}+\widetilde{b}^{\langle k\rangle}+I_{2} \leq n, s_{j}<I_{2}, \xi(n)=1\right)$

$$
\begin{aligned}
+\sum_{k=1}^{n} e_{k} \sum_{j=1}^{\infty} h_{j} \sum_{l=1}^{j} P\left(I_{1}+\widetilde{b}^{\langle k\rangle}+I_{2} \leq n,\right. \\
\left.s_{l-1}<I_{2} \leq s_{l-1}+W_{l}, \xi(n)=1\right)
\end{aligned}
$$

$+\sum_{k=1}^{n} e_{k} \sum_{j=1}^{\infty} h_{j} \sum_{l=1}^{j} \sum_{i=1}^{\infty} P\left(s_{l-1}+W_{l}<I_{2} \leq s_{l}\right.$,

$$
\begin{aligned}
& I_{2}+\delta_{i-1}<s_{l} \leq I_{2}+\delta_{i}, \\
& \left.n \geq I_{1}+\tilde{b}^{\langle k\rangle}+s_{l}, \xi(n)=1\right)
\end{aligned}
$$

$=\sum_{k=1}^{\infty} e_{k} \sum_{i=1}^{n} P\left(I_{1}=i\right)\left[1-\sum_{j=k}^{n-i} P\left(\widetilde{b}^{\langle k\rangle}=j\right)\right]$

$+\sum_{k=1}^{n} e_{k} \sum_{j=1}^{\infty} h_{j} \sum_{m=1}^{\infty} e_{m}$

$\times \sum_{u=k+1}^{n} \sum_{r=1}^{n-u} P\left(I_{1}+b^{\langle k\rangle}=u\right) A_{m}(n-u-r)$

$\times P\left(I_{2}=r\right) \sum_{w=2 j-2}^{r-1} P\left(s_{j}=w\right)$

$+\sum_{k=1}^{n} e_{k} \sum_{j=1}^{\infty} h_{j} \sum_{l=1}^{j} \sum_{m=1}^{\infty} e_{m}$

$\times \sum_{u=k+1}^{n} \sum_{r=1}^{n-u} P\left(I_{1}+b^{\langle k\rangle}=u\right) P\left(I_{2}=r\right) A_{m}(n-u-r)$

$\times\left[\sum_{w=2 l-2}^{r-1} P\left(s_{l-1}=w\right)-\sum_{w=2 l-1}^{r-1} P\left(s_{l-1}+W_{l}=w\right)\right]$ 


$$
\begin{aligned}
& +\sum_{k=1}^{n} e_{k} \sum_{j=1}^{\infty} h_{j} \sum_{l=1}^{j} \sum_{i=1}^{\infty} \sum_{m[i]=i}^{\infty} e_{m_{1}} \cdots e_{m_{i}} \\
& \times \sum_{u=k+1}^{n} \sum_{t=2 l-1}^{n-u} \sum_{r=1}^{n-u-t} A_{m[i]}(n-u-t-r) \\
& \times P\left(I_{1}+b^{\langle k\rangle}=u\right) P\left(s_{l-1}+W_{l}=t\right) P\left(V_{l}=r\right) \\
& \times C_{r}^{i} p^{i}(1-p)^{r-i}(1-p)^{t} .
\end{aligned}
$$

By the same way, for $i \geq 1$, we have

$$
\begin{aligned}
A_{i}(n)= & 1-\sum_{j=i}^{n} P\left(\widetilde{b}^{\langle i\rangle}=j\right) \sum_{j=1}^{\infty} h_{j} \sum_{m=1}^{\infty} e_{m} \sum_{u=i}^{n} \sum_{r=1}^{n-u} P\left(\widetilde{b}^{\langle i\rangle}=u\right) \\
& \times P\left(I_{1}=r\right) A_{m}(n-u-r) \sum_{w=2 j}^{r-1} P\left(s_{j}=w\right) \\
& +\sum_{k=1}^{n} e_{k} \sum_{j=1}^{\infty} h_{j} \sum_{l=1}^{j} \sum_{m=1}^{\infty} e_{m} \\
& \times \sum_{u=i r=1}^{n} \sum^{n-u} P\left(b^{\langle i\rangle}=u\right) A_{m}(n-u-r) \\
& \times P\left(I_{1}=r\right) \\
& \times\left[\sum_{w=2 l-2}^{r-1} P\left(s_{l-1}=w\right)-\sum_{w=2 l-1}^{r-1} P\left(s_{l-1}+W_{l}=w\right)\right] \\
& +\sum_{j=1}^{\infty} h_{j} \sum_{l=1}^{j} \sum_{i=1}^{\infty} \sum_{m[i]=i}^{\infty} e_{m_{1}} \ldots e_{m_{i}} \\
& \times C_{r}^{i} p^{i}(1-p)^{r-i}(1-p)^{t} . \\
& \left.\times \sum_{u=i}^{n-u} \sum_{t=2 l-1}^{n-u-t} A_{r=1}^{\langle i\rangle}=u\right) P\left(s_{l-1}+W_{l}=t\right) P\left(V_{l}=r\right) \\
& \times(n-t-r)
\end{aligned}
$$

Taking $z$-transforms of (9) and (10), respectively, we get

$$
\begin{aligned}
a_{0}(z)= & \frac{\tau(z)}{1-z}[1-A(\widetilde{b}(z))] \\
& +[\tau(z)]^{2} A(\widetilde{b}(z)) H(\theta(z)) \sum_{m=1}^{\infty} e_{m} a_{m}(z) \\
& +[\tau(z)]^{2} A(\widetilde{b}(z))[1-w(\bar{p} z)] \\
& \times \frac{1-H(\theta(z))}{1-\theta(z)} \sum_{m=1}^{\infty} e_{m} a_{m}(z) \\
& +\tau(z) A(\widetilde{b}(z)) w(\bar{p} z) \frac{1-H(\theta(z))}{1-\theta(z)} \\
& \times \sum_{i=1}^{\infty} \sum_{m[i]=i}^{\infty} e_{m_{1}} \cdots e_{m_{i}} a_{m[i]}(z) \sum_{r=1}^{\infty} v_{r} z^{r} C_{r}^{i} p^{i}(1-p)^{r-i}
\end{aligned}
$$

$$
\begin{aligned}
a_{i}(z)= & \frac{1-\widetilde{b}^{i}(z)}{1-z}+\tau(z) \widetilde{b}^{i}(z) H(\theta(z)) \sum_{m=1}^{\infty} e_{m} a_{m}(z) \\
& +\tau(z) \widetilde{b}^{i}(z)[1-w(\bar{p} z)] \frac{1-H(\theta(z))}{1-\theta(z)} \sum_{m=1}^{\infty} e_{m} a_{m}(z) \\
& +\widetilde{b}^{i}(z) w(\bar{p} z) \frac{1-H(\theta(z))}{1-\theta(z)} \\
& \times \sum_{i=1}^{\infty} \sum_{m[i]=i}^{\infty} e_{m_{1}} \cdots e_{m_{i}} a_{m[i]}(z) \\
& \times \sum_{r=1}^{\infty} v_{r} z^{r} C_{r}^{i} p^{i}(1-p)^{r-i}, \quad i \geq 1 .
\end{aligned}
$$

It follows from (11) and (12) that

$$
a_{i}(z)=\frac{1}{1-z}-\frac{\widetilde{b}^{i}(z)}{1-z}\left[\frac{\tau(z)-(1-z) a_{0}(z)}{\tau(z) A(\tilde{b}(z))}\right], \quad i \geq 1
$$

Substituting (13) into (11) and noting

$$
\begin{gathered}
\sum_{i=1}^{\infty} \sum_{m[i]=i}^{\infty} e_{m_{1}} \cdots e_{m_{i}} \widetilde{b}^{m[i]}(z) \sum_{r=1}^{\infty} v_{r} z^{r} C_{r}^{i} p^{i}(1-p)^{r-i} \\
=\sum_{r=1}^{\infty} v_{r} z^{r} \sum_{i=1}^{\infty} C_{r}^{i}[p A(\widetilde{b}(z))]^{i}(1-p)^{r-i} \\
=v(\bar{p} z+p z A(\widetilde{b}(z)))-v(\bar{p} z),
\end{gathered}
$$

we easily get (4). Equation (5) is obtained by (4) and (13). By Lemmas 8 and 9, we have $\lim _{n \rightarrow \infty} A_{i}(n)=\lim _{z \uparrow 1}(1-$ $z) a_{i}(z)$. So, (6) follows by (4), (5), L' Hospital's rule, and direct calculations.

To study the unavailability and the failure number during $(0, n]$ of the service station, we first consider a classical discrete-time single-unit repairable system. Whenever the unit fails, the system breaks down. The failed unit is repaired immediately, and it is as good as new after repair. As soon as the repair of the failed unit is completed, the system starts to operate immediately. The lifetime $X$ of the unit is geometrically distributed with parameter $\alpha, 0 \leq \alpha<1$, and its repair time $Y$ obeys an arbitrary discrete-time distribution with a mean repair time $\beta$. Suppose that the unit is new at the initial time $n=0 . X$ and $Y$ are mutually independent. For $n \geq 0$, let

$$
\begin{gathered}
\Phi(n)=P(\text { the system is repaired at time } n), \\
M(n)=E(\text { the failure number of } \\
\text { the system during }(0, n]),
\end{gathered}
$$

and $\varphi(z)=\sum_{n=0}^{\infty} \Phi(n) z^{n}, m(z)=\sum_{n=0}^{\infty} M(n) z^{n},|z|<1$.

Similar to the discussions in a continuing-time singleunit system [16], we have the following lemma. 
Lemma 11. For $|z|<1$, then

$$
\begin{aligned}
& \varphi(z)=\frac{z \alpha[1-y(z)]}{(1-z)[1-z+z \alpha(1-y(z))]}, \\
& m(z)=\frac{z \alpha}{(1-z)[1-z+z \alpha(1-y(z))]}, \\
& \lim _{n \rightarrow \infty} \Phi(n)=\lim _{z \uparrow 1}(1-z) \varphi(z)=\frac{\alpha \beta}{1+\alpha \beta}, \\
& \lim _{n \rightarrow \infty} \frac{M(n)}{n}=\lim _{z \uparrow 1}(1-z)^{2} m(z)=\frac{\alpha}{1+\alpha \beta},
\end{aligned}
$$

where $y(z)=\sum_{j=0}^{\infty} P(Y=j) z^{j},|z|<1$.

Remark 12. Let $M_{f}(k)=M(k)-M(k-1), k \geq 1$, and let $m_{f}(z)=\sum_{n=0}^{\infty} M_{f}(n) z^{n},|z|<1$, and then according to Lemmas 8 and 9 , we have

$$
\lim _{n \rightarrow \infty} \frac{M(n)}{n}=\lim _{z \uparrow 1}(1-z) m_{f}(z)=\lim _{z \uparrow 1}(1-z)^{2} m(z) .
$$

3.2. The Unavailability of the Service Station. The unavailability of the service station at time $n$, that is, the probability that the service station is repaired at time $n$.

Theorem 13. Let $\Phi_{i}(n)=P$ (the service station is repaired at time $n \mid N(0)=i), i \geq 0$, and let $\varphi_{i}(z)=\sum_{n=0}^{\infty} \Phi_{i}(n) z^{n},|z|<$ 1 , and then the PGF of $\Phi_{i}(n)$ is

$$
\varphi_{i}(z)=\varphi(z)\left[(1-z) a_{i}(z)\right], \quad i \geq 0,
$$

and the steady-state unavailability of the service station is given by

$$
\lim _{n \rightarrow \infty} \Phi_{i}(n)=\lim _{z \uparrow 1}(1-z) \varphi_{i}(z)= \begin{cases}\operatorname{pe\mu \alpha \beta ,} & \tilde{\rho}<1 \\ \frac{\alpha \beta}{1+\alpha \beta}, & \tilde{\rho} \geq 1,\end{cases}
$$

where $\varphi(z), a_{i}(z), i \geq 0$, and $\tilde{\rho}$ are given by Lemma 11, Theorem 10, and Lemma 6, respectively.

Proof. (i) Let $\eta(n)=1$ denote the event that the service station is repaired at time $n$. The service station is repaired at time $n$ if and only if the time $n$ is during one server busy period, and the service station is repaired at time $n$.
Therefore, by total probability decomposition and renewal point technique, $\Phi_{0}(n)$ is decomposed as

$$
\begin{aligned}
& \Phi_{0}(n)=\sum_{k=1}^{\infty} e_{k} P\left(I_{1} \leq n<I_{1}+\widetilde{b}^{\langle k\rangle}, \eta(n)=1\right) \\
& +\sum_{k=1}^{n} e_{k} \sum_{j=1}^{\infty} h_{j} P\left(I_{1}+\widetilde{b}^{\langle k\rangle}+I_{2} \leq n, s_{j}<I_{2}, \eta(n)=1\right) \\
& +\sum_{k=1}^{n} e_{k} \sum_{j=1}^{\infty} h_{j} \sum_{l=1}^{j} P\left(I_{1}+\widetilde{b}^{\langle k\rangle}+I_{2} \leq n,\right. \\
& \left.s_{l-1}<I_{2} \leq s_{l-1}+W_{l}, \eta(n)=1\right) \\
& +\sum_{k=1}^{n} e_{k} \sum_{j=1}^{\infty} h_{j} \sum_{l=1}^{j} \sum_{i=1}^{\infty} P\left(s_{l-1}+W_{l} \leq I_{2} \leq s_{l},\right. \\
& I_{2}+\delta_{i-1}<s_{l} \leq I_{2}+\delta_{i} \\
& \left.n \geq I_{1}+\widetilde{b}^{\langle k\rangle}+s_{l}, \eta(n)=1\right) \\
& =\sum_{k=1}^{\infty} e_{k} \sum_{i=1}^{n} P\left(I_{1}=i\right) S_{k}(n-i) \\
& +\sum_{k=1}^{n} e_{k} \sum_{j=1}^{\infty} h_{j} \sum_{m=1}^{\infty} e_{m} \sum_{u=k+1}^{n} \sum_{r=1}^{n-u} P\left(I_{1}+b^{\langle k\rangle}=u\right) \\
& \times \Phi_{m}(n-u-r) P\left(I_{2}=r\right) \sum_{w=2 j}^{r-1} P\left(s_{j}=w\right) \\
& +\sum_{k=1}^{n} e_{k} \sum_{j=1}^{\infty} h_{j} \sum_{l=1}^{j} \sum_{m=1}^{\infty} e_{m} \\
& \times \sum_{u=k+1}^{n} \sum_{r=1}^{n-u} P\left(I_{1}+b^{\langle k\rangle}=u\right) P\left(I_{2}=r\right) \Phi_{m}(n-u-r) \\
& \times\left[\sum_{w=2 l-2}^{r-1} P\left(s_{l-1}=w\right)-\sum_{w=2 l-1}^{r-1} P\left(s_{l-1}+W_{l}=w\right)\right] \\
& +\sum_{k=1}^{n} e_{k} \sum_{j=1}^{\infty} h_{j} \sum_{l=1}^{j} \sum_{i=1}^{\infty} \sum_{m[i]=i}^{\infty} e_{m_{1}} \cdots e_{m_{i}} \\
& \times \sum_{u=k+1}^{n} \sum_{t=2 l-1}^{n-u} \sum_{r=1}^{n-u-t} \Phi_{m[i]}(n-u-t-r) \\
& \times P\left(I_{1}+b^{\langle k\rangle}=u\right) P\left(s_{l-1}+W_{l}=t\right) P\left(V_{l}=r\right) \\
& \times C_{r}^{i} p^{i}(1-p)^{r-i}(1-p)^{t}
\end{aligned}
$$

where $S_{k}(n)=P\left(0 \leq n<\widetilde{b}^{\langle k\rangle}\right.$, and the service station is repaired at time $n), k \geq 1$. 
Similarly, for $i \geq 1$, we have the decomposition of $\Phi_{i}(n)$ as follows:

$$
\begin{aligned}
& \Phi_{i}(n)=S_{i}(n)+\sum_{j=1}^{\infty} h_{j} \sum_{m=1}^{\infty} e_{m} \\
& \times \sum_{u=i}^{n} \sum_{r=1}^{n-u} P\left(\widetilde{b}^{\langle i\rangle}=u\right) \Phi_{m}(n-u-r) P\left(I_{1}=r\right) \\
& \times \sum_{w=2 j}^{r-1} P\left(s_{j}=w\right)+\sum_{j=1}^{\infty} h_{j} \sum_{l=1}^{j} \sum_{m=1}^{\infty} e_{m} \\
& \times \sum_{u=i}^{n} \sum_{r=1}^{n-u} P\left(b^{\langle i\rangle}=u\right) \Phi_{m}(n-u-r) \\
& \times P\left(I_{1}=r\right) \\
& \times\left[\sum_{w=2 l-2}^{r-1} P\left(s_{l-1}=w\right)-\sum_{w=2 l-1}^{r-1} P\left(s_{l-1}+W_{l}=w\right)\right] \\
& +\sum_{j=1}^{\infty} h_{j} \sum_{l=1}^{j} \sum_{i=1}^{\infty} \sum_{m[i]=i}^{\infty} e_{m_{1}} \cdots e_{m_{i}} \\
& \times \sum_{u=i}^{n} \sum_{t=2 l-1}^{n-u} \sum_{r=1}^{n-u-t} \Phi_{m[i]}(n-u-t-r) \\
& \times P\left(b^{\langle i\rangle}=u\right) P\left(s_{l-1}+W_{l}=t\right) P\left(V_{l}=r\right) \\
& \times C_{r}^{i} p^{i}(1-p)^{r-i}(1-p)^{t} .
\end{aligned}
$$

(ii) For $i \geq 1$,

$$
\begin{aligned}
& S_{i}(n)=\Phi(n)-\sum_{k=i}^{n} P\left(\widetilde{b}^{\langle i\rangle}=k\right) \Phi(n-k), \\
& \sum_{n=0}^{\infty} S_{i}(n) z^{n}=\varphi(z)\left[1-\widetilde{b}^{i}(z)\right], \quad|z|<1,
\end{aligned}
$$

where $\Phi(n)$ and $\varphi(z)$ are given by Lemma 11 .

In fact, $\Phi(n)$ can be decomposed as

$$
\begin{aligned}
\Phi(n)= & P\left(\text { the system is repaired at time } n, \widetilde{b}^{\langle i\rangle} \leq n\right) \\
& +P\left(\text { the system is repaired at time } n, \widetilde{b}^{\langle i\rangle}>n\right) \\
= & \sum_{k=i}^{n} P\left(\widetilde{b}^{\langle i\rangle}=k\right) \Phi(n-k)+S_{i}(n) .
\end{aligned}
$$

So, (22) is easily obtained. (iii) Taking $z$-transforms of (20) and (21), respectively, and by means of (22), we have the following results:

$$
\begin{aligned}
\varphi_{0}(z)= & \tau(z) \varphi(z)[1-A(\widetilde{b}(z))] \\
& +[\tau(z)]^{2} A(\widetilde{b}(z)) H(\theta(z)) \sum_{m=1}^{\infty} e_{m} \varphi_{m}(z) \\
& +[\tau(z)]^{2} A(\widetilde{b}(z))[1-w(\bar{p} z)] \frac{1-H(\theta(z))}{1-\theta(z)} \\
& \times \sum_{m=1}^{\infty} e_{m} \varphi_{m}(z) \\
& +\tau(z) A(\widetilde{b}(z)) w(\bar{p} z) \frac{1-H(\theta(z))}{1-\theta(z)} \\
& \times \sum_{i=1}^{\infty} \sum_{m[i]=i}^{\infty} e_{m_{1}} \cdots e_{m_{i}} \varphi_{m[i]}(z) \\
& \times \sum_{r=1}^{\infty} v_{r} z^{r} C_{r}^{i} p^{i}(1-p)^{r-i}
\end{aligned}
$$

$$
\begin{aligned}
\varphi_{i}(z)= & \varphi(z)\left[1-\widetilde{b}^{i}(z)\right]+\tau(z) \widetilde{b}^{i}(z) H(\theta(z)) \sum_{m=1}^{\infty} e_{m} \varphi_{m}(z) \\
& +\tau(z) \widetilde{b}^{i}(z)[1-w(\bar{p} z)] \frac{1-H(\theta(z))}{1-\theta(z)} \sum_{m=1}^{\infty} e_{m} \varphi_{m}(z) \\
& +\widetilde{b}^{i}(z) w(\bar{p} z) \frac{1-H(\theta(z))}{1-\theta(z)} \\
& \times \sum_{i=1}^{\infty} \sum_{m[i]=i}^{\infty} e_{m_{1}} \cdots e_{m_{i}} \varphi_{m[i]}(z)
\end{aligned}
$$$$
\times \sum_{r=1}^{\infty} v_{r} z^{r} C_{r}^{i} p^{i}(1-p)^{r-i}, \quad i \geq 1
$$

Performing similar operations in the proof of Theorem 10 on (24), we can complete the proof by Theorem 10 and Lemma 11 above.

3.3. The Mean Failure Number of Service Station during $(0, n]$

Theorem 14. Let $M_{i}(n)=E$ (the failure number of service station during $(0, n] \mid N(0)=i), i \geq 0$, and let $m_{i}(z)=$ $\sum_{n=0}^{\infty} M_{i}(n) z^{n},|z|<1$, and then the PGF of $M_{i}(n)$ is

$$
m_{i}(z)=m(z)\left[(1-z) a_{i}(z)\right], \quad i \geq 0,
$$

and the steady-state failure frequency of service station is given by

$$
\lim _{n \rightarrow \infty} \frac{M_{i}(n)}{n}=\lim _{z \uparrow 1}(1-z)^{2} m_{i}(z)= \begin{cases}\text { pe } \mu \alpha, & \tilde{\rho}<1 \\ \frac{\alpha}{1+\alpha \beta}, & \tilde{\rho} \geq 1\end{cases}
$$


where $m(z), a_{i}(z), i \geq 0$, and $\tilde{\rho}$ are given by Lemma 11, Theorem 10, and Lemma 6 , respectively.

Proof. (1) For $i \geq 1$, let

$J_{i}(n)=E\left(0 \leq n<\tilde{b}^{\langle i\rangle}\right.$, the failure number of service station during $(0, n])$, $L_{i}(n)=E\left(\widetilde{b}^{\langle i\rangle} \leq n\right.$, the failure number of service station during $\left.\left(0, \widetilde{b}^{\langle i\rangle}\right]\right)$.

Then, similar to (22) above, we have

$$
\begin{gathered}
J_{i}(n)+L_{i}(n)=M(n)-\sum_{k=i}^{n} P\left(\widetilde{b}^{\langle i\rangle}=k\right) M(n-k), \quad i \geq 1, \\
\sum_{n=0}^{\infty}\left[J_{i}(n)+L_{i}(n)\right] z^{n}=m(z)\left[1-\widetilde{b}^{i}(z)\right], \quad|z|<1,
\end{gathered}
$$

where $M(n)$ and $m(z)$ are given by Lemma 11 .

(2) Using total probability decomposition and renewal point technique, we have the decomposition of $M_{0}(n)$ as follows:

$$
\begin{gathered}
M_{0}(n) \\
=\sum_{k=1}^{\infty} e_{k}\left\{E \left(I_{1} \leq n<I_{1}+\widetilde{b}^{\langle k\rangle}\right.\right. \text {, the failure number of } \\
\text { service station during } \left.\left(I_{1}, n\right]\right) \\
+E\left(n \geq I_{1}+\widetilde{b}^{\langle k\rangle}\right. \text {, the failure number of } \\
\text { service station during } \left.\left.\left(I_{1}, I_{1}+\widetilde{b}^{\langle k\rangle}\right]\right)\right\} \\
+\sum_{k=1}^{n} e_{k} \sum_{j=1}^{\infty} h_{j} E\left(I_{1}+\widetilde{b}^{\langle k\rangle}+I_{2} \leq n, s_{j}<I_{2},\right.
\end{gathered}
$$

the failure number of service station during

$$
\left.\left(I_{1}+\widetilde{b}^{\langle k\rangle}+I_{2}, n\right]\right)
$$

$$
+\sum_{k=1}^{n} e_{k} \sum_{j=1}^{\infty} h_{j}
$$

$$
\times \sum_{l=1}^{j} E\left(s_{l-1}<I_{2} \leq s_{l-1}+W_{l}, I_{1}+\widetilde{b}^{\langle k\rangle}+I_{2} \leq n,\right.
$$

the failure number of service station during

$$
\left.\left(I_{1}+\widetilde{b}^{\langle k\rangle}+I_{2}, n\right]\right)
$$

$$
\begin{aligned}
& +\sum_{k=1}^{n} e_{k} \sum_{j=1}^{\infty} h_{j} \\
& \times \sum_{l=1}^{j} \sum_{i=1}^{\infty} P\left(s_{l-1}+W_{l} \leq I_{2} \leq s_{l}, I_{2}+\delta_{i-1}<s_{l} \leq I_{2}+\delta_{i},\right.
\end{aligned}
$$
$n \geq I_{1}+\widetilde{b}^{\langle k\rangle}+s_{l}$, the failure number of service station during $\left.\left(I_{1}+\widetilde{b}^{\langle k\rangle}+s_{l}, n\right]\right)$

$$
\begin{aligned}
= & \sum_{k=1}^{\infty} e_{k} \sum_{i=1}^{n} P\left(I_{1}=i\right)\left[J_{k}(n-i)+L_{k}(n-i)\right] \\
& +\sum_{k=1}^{n} e_{k} \sum_{j=1}^{\infty} h_{j} \sum_{m=1}^{\infty} e_{m} \\
& \times \sum_{u=k+1}^{n} \sum_{r=1}^{n-u} P\left(I_{1}+b^{\langle k\rangle}=u\right) M_{m}(n-u-r) P\left(I_{2}=r\right) \\
& \times \sum_{w=2}^{r-1} P\left(s_{j}=w\right)+\sum_{k=1}^{n} e_{k} \sum_{j=1}^{\infty} h_{j} \sum_{l=1}^{j} \sum_{m=1}^{\infty} e_{m} \\
& \times \sum_{u=k+1}^{n} \sum_{r=1}^{n-u} P\left(I_{1}+b^{\langle k\rangle}=u\right) \\
& \times P\left(I_{2}=r\right) M_{m}(n-u-r) \\
& \times\left[\sum_{w=2 l-2}^{r-1} P\left(s_{l-1}=w\right)-\sum_{w=2 l-1}^{r-1} P\left(s_{l-1}+W_{l}=w\right)\right] \\
& \times C_{r}^{i} p^{i}(1-p)^{r-i}(1-p)^{t} . \\
& +\sum_{k=1}^{n} e_{k} \sum_{j=1}^{\infty} h_{j} \sum_{l=1}^{j} \sum_{i=1}^{\infty} \sum_{m[i]=i}^{\infty} e_{m_{1}} \cdots e_{m_{i}}+\sum_{t=2 l-1}^{\langle k-u} \sum_{r=1}^{n-u-t} M_{m[i]}(n-u-t-r) \\
& \times\left(s_{l-1}+W_{l}=t\right) P\left(V_{l}=r\right)
\end{aligned}
$$

Similarly, $M_{i}(n), i \geq 1$ are decomposed as

$$
\begin{aligned}
M_{i}(n)= & J_{i}(n)+L_{i}(n)+\sum_{j=1}^{\infty} h_{j} \sum_{m=1}^{\infty} e_{m} \\
& \times \sum_{u=i}^{n} \sum_{r=1}^{n-u} P\left(\widetilde{b}^{\langle i\rangle}=u\right) M_{m}(n-u-r) P\left(I_{1}=r\right)
\end{aligned}
$$




$$
\begin{aligned}
& \times \sum_{w=2 j}^{r-1} P\left(s_{j}=w\right)+\sum_{j=1}^{\infty} h_{j} \sum_{l=1}^{j} \sum_{m=1}^{\infty} e_{m} \\
& \times \sum_{u=i}^{n} \sum_{r=1}^{n-u} P\left(b^{\langle i\rangle}=u\right) M_{m}(n-u-r) \\
& \times P\left(I_{1}=r\right) \\
& \left.\times \sum_{w=2 l-2}^{r-1} P\left(s_{l-1}=w\right)-\sum_{w=2 l-1}^{r-1} P\left(s_{l-1}+W_{l}=w\right)\right] \\
& +\sum_{j=1}^{\infty} h_{j} \sum_{l=1}^{j} \sum_{i=1}^{\infty} \sum_{m[i]=i}^{\infty} e_{m_{1}} \cdots e_{m_{i}} \\
& \times \sum_{u=i}^{n} \sum_{t=2 l-1}^{n-u} \sum_{r=1}^{n-u-t} M_{m[i]}(n-u-t-r) \\
& \times P\left(b^{\langle i\rangle}=u\right) P\left(s_{l-1}+W_{l}=t\right) P\left(V_{l}=r\right) \\
& \times C_{r}^{i} p^{i}(1-p)^{r-i}(1-p)^{t} .
\end{aligned}
$$

Taking $z$-transforms of (29) and (30), and performing similar operations in the proof of Theorem 10, we can complete the proof by Theorem 10 and Lemma 11 above.

Remark 15. Equations (18) and (25) show that studying the unavailability and the mean failure number during $(0, n]$ of the service station is equivalent to studying the corresponding indices in the classical discrete-time single-unit system and the probability that the time $n$ is during server busy period presented in this paper. It is important that (18) and (25) indicate the structures of the service station indices.

Remark 16. It is seen from (6), (19), (26), and Lemma 11 that the steady-state relation equations $\lim _{n \rightarrow \infty} \Phi_{i}(n)=\lim _{n \rightarrow \infty} \Phi(n) \lim _{n \rightarrow \infty} A_{i}(n)$ and $\lim _{n \rightarrow \infty} M_{i}(n) / n=\lim _{n \rightarrow \infty}(M(n) / n) \lim _{n \rightarrow \infty} A_{i}(n), i \geq 0$ also hold. Further, the two steady-state results are independent of the initial state $N(0)=i, i \geq 0$, and they have nothing to do with server vacations and delayed times.

Remark 17. It should be noted that (18), (25), and two steadystate relation equations derived in Remark 16 are new results, and these important reliability relation equations are not obtained by the existing discrete analytic techniques, such as the discrete supplementary variable technique [1-8] or matrix-geometric solution method $[9,10]$.

\section{Special Cases}

Case 1. If $H(z)=z^{J}$ and $P\left(W_{i}=0\right)=1, i \geq 1$, then our model becomes a discrete-time $\mathrm{Geom}^{X} / \mathrm{G} / 1$ queue with an unreliable service station and at most $J$ vacations. In this special case, for $\tilde{\rho}=p e \mu(1+\alpha \beta)<1$, the busy probabilities of the server $\lim _{n \rightarrow \infty} A_{i}(n)$, the unavailability $\lim _{n \rightarrow \infty} \Phi_{i}(n)$, and the failure frequency $\lim _{n \rightarrow \infty} M_{i}(n) / n$ are as follows:

$$
\begin{gathered}
\lim _{n \rightarrow \infty} A_{i}(n)=\operatorname{pe\mu }(1+\alpha \beta)=\tilde{\rho}, \quad \lim _{n \rightarrow \infty} \Phi_{i}(n)=\operatorname{pe\mu \alpha } \beta, \\
\lim _{n \rightarrow \infty} \frac{M_{i}(n)}{n}=\text { pe } \mu \alpha .
\end{gathered}
$$

These results are very similar to those for the continuoustime counterpart, which also have nothing to do with server vacations (see [17, Sections 3 and 4, p 9-10] and the following fact).

In fact, for the continuous-time counterpart, that is, the $\mathrm{M}^{X} / \mathrm{G} / 1$ queue with an unreliable service station and at most $J$ vacations (setting $p=0$ in Ke's model [17]), under steadystate condition $\rho_{H}<1\left(\rho_{H}=\lambda E[X] E[S](1+\alpha E[R])\right.$, the busy probability of the server $P_{\text {busy }}$, the unavailability $P_{r}$, and the failure frequency $M_{f}$ are given, respectively, by

$$
\begin{gathered}
P_{\text {busy }}=\frac{E[H]}{E[C]}=\lambda E[X] E[S](1+\alpha E[R])=\rho_{H}, \\
P_{r}=\frac{E[D]}{E[C]}=\lambda E[X] E[S] \alpha E[R], \quad M_{f}=\lambda E[X] E[S] \alpha,
\end{gathered}
$$

where

$$
E[H]=\left(\frac{\left(1-\gamma_{0}^{J}\right) \lambda E[V]}{1-\gamma_{0}}+\gamma_{0}^{J}\right)\left(\frac{E[X] E[S](1+\alpha E[R])}{1-\rho_{H}}\right)
$$

is the expected length of completion period (here, $\gamma_{0}=$ $\int_{0}^{\infty} e^{-\lambda t} d V(t)$, and $V(t)$ and $E[V]$ are the vacation distribution function and the expected length of each vacation),

$$
E[D]=\left(\frac{\left(1-\gamma_{0}^{J}\right) \lambda E[V]}{1-\gamma_{0}}+\gamma_{0}^{J}\right)\left(\frac{E[X] E[S] \alpha E[R]}{1-\rho_{H}}\right)
$$

is the expected length of breakdown period of service station,

$$
E[C]=\left(\frac{\left(1-\gamma_{0}^{J}\right) \lambda E[V]}{1-\gamma_{0}}+\gamma_{0}^{J}\right)\left(\frac{1}{\lambda\left(1-\rho_{H}\right)}\right)
$$

is the expected length of busy cycle, and $\lambda, E[X], E[S], \alpha$, and $E[R]$ are corresponding to $p, e, u, \alpha$, and $\beta$ in our model, respectively, (see [17, Sections 3 and 4, p 9-10] for details).

Case 2. If $H(z)=z, P(D=1)=1$ and $P\left(W_{i}=0\right)=1, i \geq$ 1 , then our model becomes a discrete-time Geom/G/1 queue with an unreliable service station and a single vacation. In this case, for $\tilde{\rho}=p \mu(1+\alpha \beta)<1$, we get

$$
\begin{gathered}
\lim _{n \rightarrow \infty} A_{i}(n)=p \mu(1+\alpha \beta)=\tilde{\rho}, \quad \lim _{n \rightarrow \infty} \Phi_{i}(n)=p \mu \alpha \beta, \\
\lim _{n \rightarrow \infty} \frac{M_{i}(n)}{n}=p \mu \alpha,
\end{gathered}
$$


TABLE 1: The effects of batch packet arrival rate $p$ on IP controller $(n=1, \alpha=0.25)$.

\begin{tabular}{lcccc}
\hline$p$ & $\tilde{\rho}$ & $\lim _{n \rightarrow \infty} A_{i}(n)$ & $\lim _{n \rightarrow \infty} \Phi_{i}(n)$ & $\lim _{n \rightarrow \infty}\left(M_{i}(n) / n\right)$ \\
\hline 0.10 & 0.2100 & 0.2100 & 0.0900 & 0.0300 \\
0.25 & 0.5250 & 0.5250 & 0.2250 & 0.0750 \\
0.40 & 0.8400 & 0.8400 & 0.3600 & 0.1200 \\
0.55 & 1.1550 & 1 & 0.4286 & 0.1429 \\
0.70 & 1.4700 & 1 & 0.4286 & 0.1429 \\
0.85 & 1.7850 & 1 & 0.4286 & 0.1429 \\
\hline
\end{tabular}

TABLE 2: The effects of batch size parameter $n$ on IP controller $(p=0.15, \alpha=0.25)$.

\begin{tabular}{lcccc}
\hline$n$ & $\tilde{\rho}$ & $\lim _{n \rightarrow \infty} A_{i}(n)$ & $\lim _{n \rightarrow \infty} \Phi_{i}(n)$ & $\lim _{n \rightarrow \infty}\left(M_{i}(n) / n\right)$ \\
\hline 1 & 0.3150 & 0.3150 & 0.1350 & 0.0450 \\
3 & 0.6300 & 0.6300 & 0.2700 & 0.0900 \\
5 & 0.9450 & 0.9450 & 0.4050 & 0.1350 \\
7 & 1.2600 & 1 & 0.4286 & 0.1429 \\
9 & 1.5750 & 1 & 0.4286 & 0.1429 \\
11 & 1.8900 & 1 & 0.4286 & 0.1429 \\
\hline
\end{tabular}

which are very similar to those for the continuous-time counterpart [18].

\section{Numerical Examples}

To demonstrate our model and analysis results, the switchedvirtual-connection- (SVC-) based IP-over-ATM network [19] is presented as a practical example of our model. In such a system, packets arrive in batch and can be modelled as a Bernoulli process with rate $p$. The distribution for batch size $D$ is taken as $P(D=k)=1 / n, k=1,2, \ldots, n$. The service time of each packet is assumed to follow a geometric distribution with mean $\xi=1.2$. Whenever the last packet is served, IP controller (server) releases the SVC (vacations). Before each release, the server checks the packets (delayed vacation). If no packets occur, a decision is made for another release to be performed next. Otherwise, if packets occur, service restarts. Moreover, the service may be interrupted (server breakdown) due to some unpredictable events. Suppose that unpredictable events occur according to a Bernoulli process with rate $\alpha$ and broken server is immediately recovered. The recovery time obeys negative binomial with generating function $Y(x)=(2 x /(3-x))^{2}$ with mean $\beta=3$. The service will continuously start when the interruption is recovered.

Several numerical results in Tables 1-3 analyze the effects of ATM network parameters on IP controller (server) performance, including the busy probability of IP controller $\lim _{t \rightarrow \infty} A_{i}(n)$, the unavailability $\lim _{t \rightarrow \infty} \Phi_{i}(n)$, and the failure frequency $\lim _{t \rightarrow \infty} M(n) / n$. In addition, the system load $\tilde{\rho}$ is also considered.

With analysis results in Section 3, the effects of batch packet arrival rate $p$ on IP controller indices are reported in Table 1 , where we set $(n, \alpha)=(1,0.25)$. It is seen from Table 1 that as $p$ increases, four performance indices all increase, but when $p \geq 0.55$, the system load $\tilde{\rho}$ is more than one, and the other three indices do not change. This is because the queueing system becomes unstable. Table 2 shows the effects of batch size on IP controller indices, where we set $(p, \alpha)=(0.15,0.25)$. We observe that the influence of batch size on four indices is very similar to that of $p$. The effects of unpredictable events arrival rate $\alpha$ on IP controller indices are shown in Table 3 for the set of parameters $(p, n)=(0.15,1)$. We note that all IP controller indices increase monotonously as the value $\alpha$ increases. Moreover, as $\alpha \geq 1.65$, IP controller is always busy.

Finally, we conclude that batch packet arrival, batch size, and unpredictable events arrival affect IP controller performance under the stability condition; that is, $\widetilde{\rho}<1$. As $\tilde{\rho} \geq 1$, IP controller indices will not be affected by batch packet arrival and batch size, and IP controller is always busy. This phenomenon is due to the unstability property of the ATM network.

\section{Conclusions}

In this paper, we use Tang's decomposition technique for reliability indices of a discrete-time repairable Geom ${ }^{X} / \mathrm{G} / 1$ queue, in which the server takes exhaustive service and multiple adaptive delayed vacation discipline, and the service station is subject to failures and repairs. According to such a novel analytic technique, we discuss main reliability indices of the service station, such as the probability that the time $n$ is during server busy period, the unavailability and the average failure number during $(0, n]$. Also, we derive some important reliability relation equations, which indicate the structures of the service station indices. It should be noted that these reliability relation equations are new results and are not obtained by existing discrete analytic techniques, such as the discrete supplementary variable technique [1-8] or matrixgeometric solution method $[9,10]$. Some special cases and numerical examples validate the derived results and show that 
TABLE 3: The effects of unpredictable events arrival rate $\alpha$ on IP controller $(p=0.15, n=1)$.

\begin{tabular}{lcccc}
\hline$\alpha$ & $\tilde{\rho}$ & $\lim _{n \rightarrow \infty} A_{i}(n)$ & $\lim _{n \rightarrow \infty} \Phi_{i}(n)$ & $\lim _{n \rightarrow \infty}\left(M_{i}(n) / n\right)$ \\
\hline 0.25 & 0.3150 & 0.3150 & 0.1350 & 0.0450 \\
0.45 & 0.4230 & 0.4770 & 0.2430 & 0.0810 \\
0.65 & 0.5310 & 0.5310 & 0.3510 & 0.1170 \\
0.85 & 0.6390 & 0.6390 & 0.4590 & 0.1530 \\
1.05 & 0.7470 & 0.7470 & 0.5670 & 0.1890 \\
1.25 & 0.8550 & 0.8550 & 0.6750 & 0.2250 \\
1.45 & 0.9630 & 0.9630 & 0.7830 & 0.2610 \\
1.65 & 1.0710 & 1 & 0.8319 & 0.2773 \\
1.85 & 1.1790 & 1 & 0.8473 & 0.2824 \\
\hline
\end{tabular}

Tang's analytic technique is applicable to reliability analysis of complex discrete-time repairable bulk arrival queues.

\section{Acknowledgments}

The authors would like to thank the referees and editor for their valuable comments and suggestions. This work is supported by the Youth Foundation of Chongqing University of Technology of China (No. 2010ZQ14) and the National Natural Science Foundation of China (No. 71171138).

\section{References}

[1] J. Wang and Q. Zhao, "Discrete-time Geo/G/1 retrial queue with general retrial queue with general retrial times and starting failures," Mathematical and Computer Modelling, vol. 45, no. 7-8, pp. 853-863, 2007.

[2] J. Wang and Q. Zhao, "A discrete-time Geo/G/1 retrial queue with starting failures and second optional service," Computers \& Mathematics with Applications, vol. 53, no. 1, pp. 115-127, 2007.

[3] I. Atencia, I. Fortes, and S. Sánchez, "A discrete-time retrial queueing system with starting failures, Bernoulli feedback and general retrial times," Computers and Industrial Engineering, vol. 57, no. 4, pp. 1291-1299, 2009.

[4] J. Wang and P. Zhang, "A discrete-time retrial queue with negative customers and unreliable server," Computers and Industrial Engineering, vol. 56, no. 4, pp. 1216-1222, 2009.

[5] P. Moreno, "A discrete-time retrial queue with unreliable server and general server lifetime," Journal of Mathematical Sciences, vol. 132, no. 5, pp. 643-655, 2006.

[6] I. Atencia and P. Moreno, "A discrete-time retrial queue with server breakdown," Asia-Pacific Journal of Operational Research, vol. 23, no. 2, pp. 247-271, 2006.

[7] Z. M. Liu and S. Gao, "Reliability indices of a Geo/G/1/1 Erlang loss system with active breakdowns under Bernoulli schedule," International Journal of Management Science and Engineering Management, vol. 5, no. 6, pp. 433-438, 2010.

[8] Y. H. Tang, M. M. Yu, and C. L. Li, “Geom/G1, G2/1/1 repairable Erlang loss system with catastrophe and second optional service," Journal of Systems Science and Complexity, vol. 24, no. 3, pp. 554-564, 2011.

[9] H. B. Yu, "The discrete-time repairable queue MAP/geometric (geometric/PH)/1," Hong Kong: Clobal-Link, pp. 1044-1050, 2000 .
[10] H.-b. Yu, Z.-k. Nie, and J.-w. Yang, "The MAP/PH/(PH/PH)/1 discrete-time queuing system with repairable server," Chinese Quarterly Journal of Mathematics, vol. 16, no. 2, pp. 59-63, 2001.

[11] Y. H. Tang, X. Yun, and S. J. Huang, "Discrete-time Geo ${ }^{X} / G / 1$ queue with unreliable server and multiple adaptive delayed vacations," Journal of Computational and Applied Mathematics, vol. 220, no. 1-2, pp. 439-455, 2008.

[12] Y. H. Tang, M. M. Yu, X. Yun, and S. J. Huang, "Reliability indices of discrete-time $\mathrm{Geo}^{X} / \mathrm{G} / 1$ queueing system with unreliable service station and multiple adaptive delayed vacations," Journal of Systems Science and Complexity, vol. 25, no. 6, pp. 1122-1135, 2012.

[13] J. J. Hunter, Mathematical Techniques of Applied Probability, Vol. II, Discrete Time Models: Techniques and Applications, Academic Press, New York, NY, USA, 1983.

[14] N. S. Tian and Z. G. Zhang, Vacation Queueing Models-Theory and Applications, Springer, New York, NY, USA, 2006.

[15] S. W. Fuhrmann and R. B. Cooper, "Stochastic decompositions in the M/G/1 queue with generalized vacations," Operations Research, vol. 33, no. 5, pp. 1117-1129, 1985.

[16] J. H. Cao and K. Cheng, Introduction to Reliability Mathematics, Higher Education Press, Beijing, China, 1986.

[17] J.-C. Ke, K.-B. Huang, and W. L. Pearn, "The performance measures and randomized optimization for an unreliable server $\mathrm{M}^{[x]} / \mathrm{G} / 1$ vacation system," Applied Mathematics and Computation, vol. 217, no. 21, pp. 8277-8290, 2011.

[18] Y. H. Tang and X. W. Tang, Queueing Theories-Foundations and Analysis Techniques, Science Press, Beijing, China, 2006.

[19] Z. Niu, Y. Takahashi, and N. Endo, "Performance evaluation of SVC-based IP-over-ATM networks," IEICE Transactions on Communications, vol. E81-B, no. 5, pp. 948-955, 1998. 


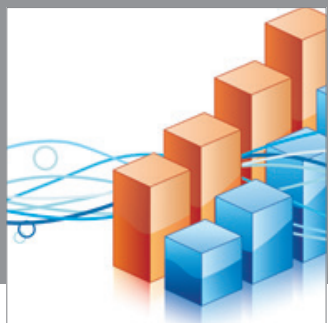

Advances in

Operations Research

mansans

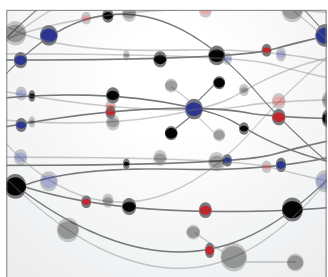

The Scientific World Journal
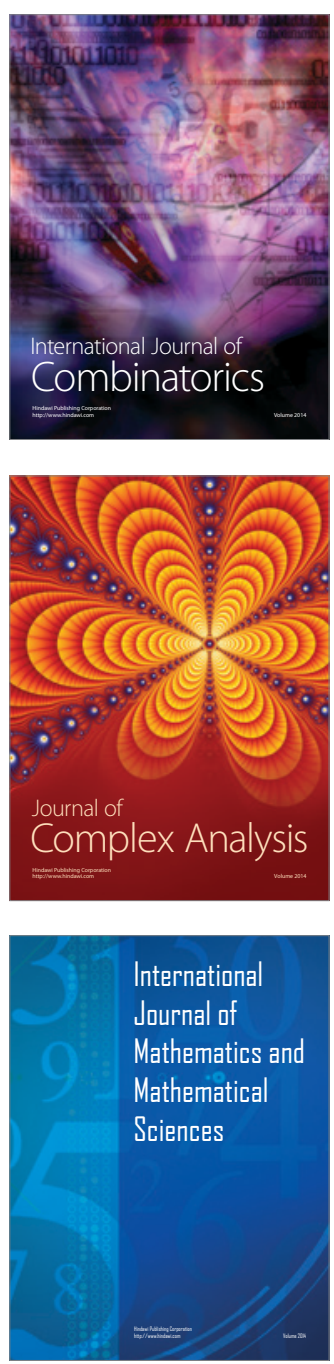
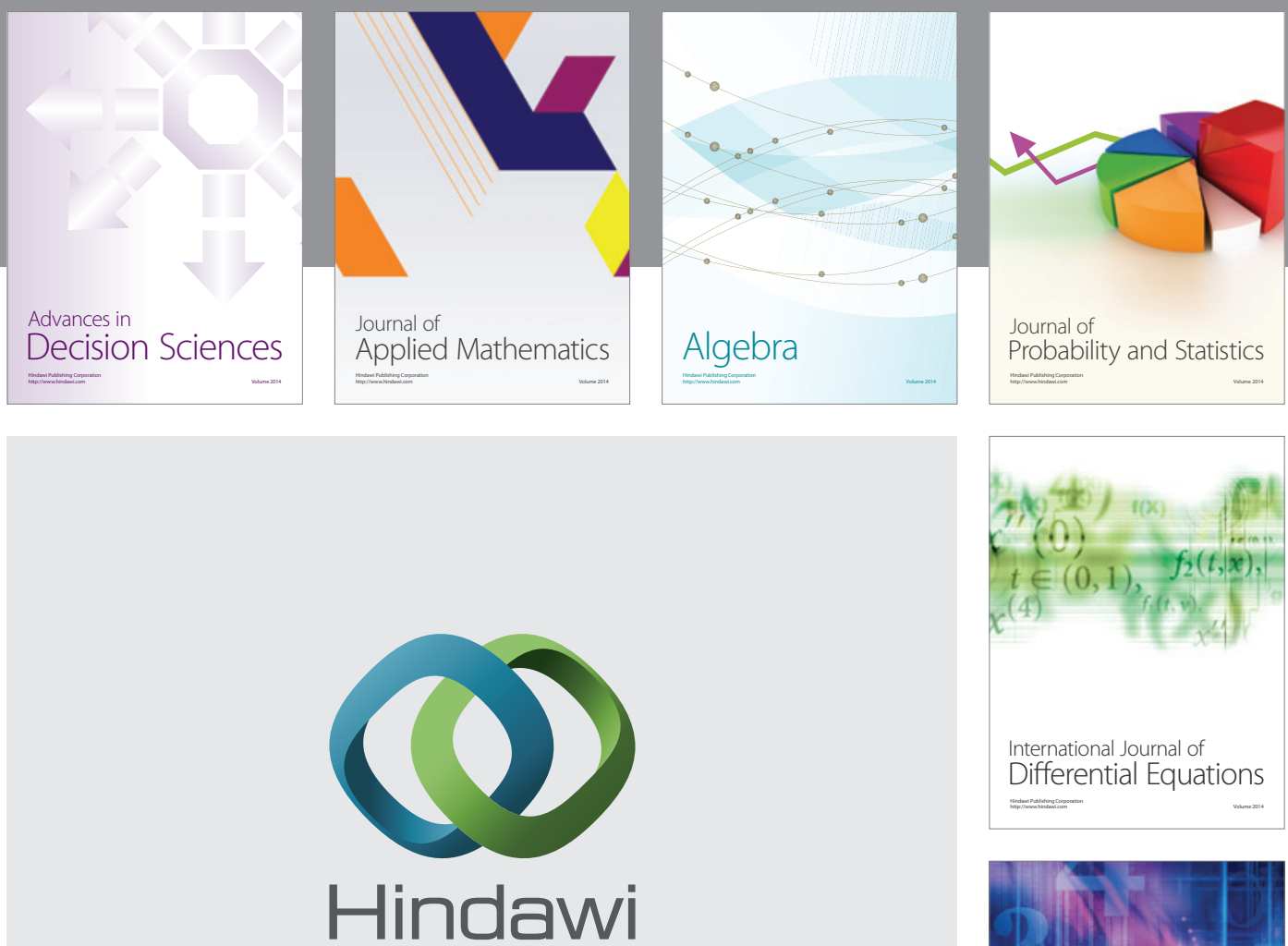

Submit your manuscripts at http://www.hindawi.com
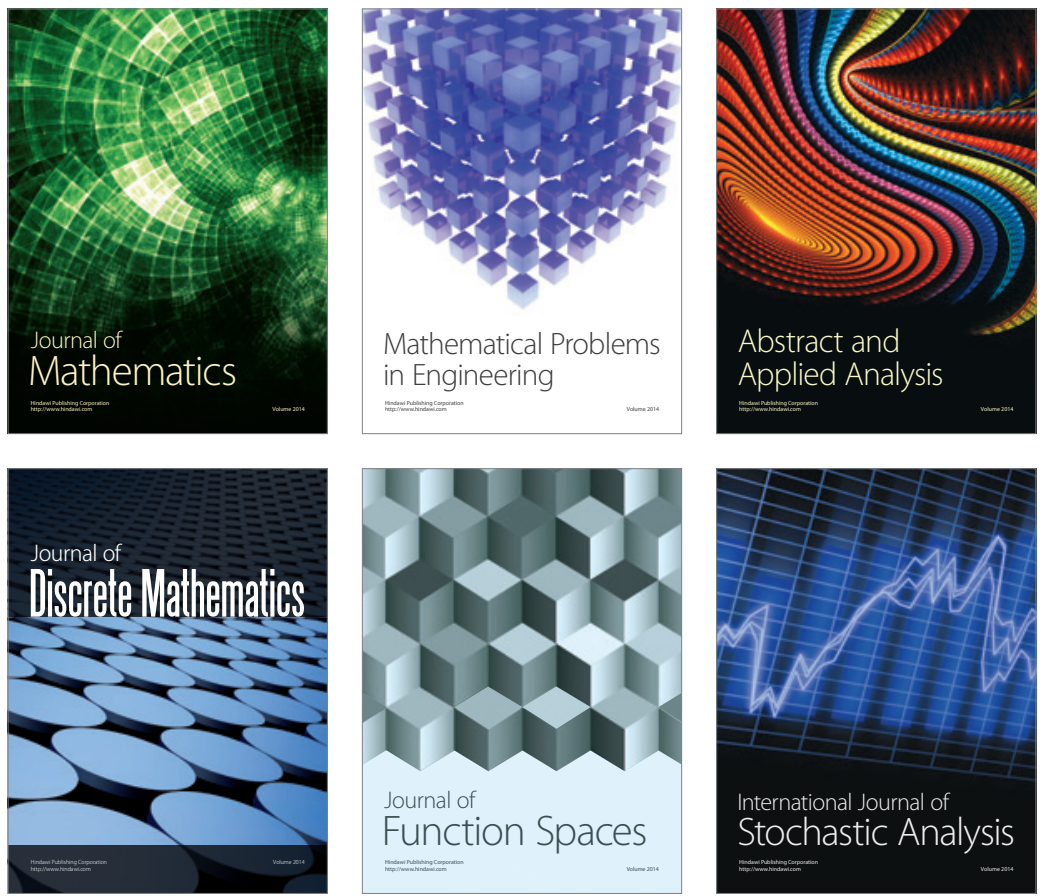

Journal of

Function Spaces

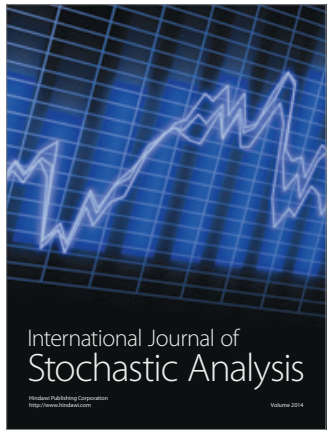

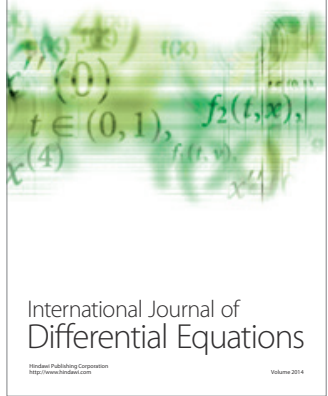
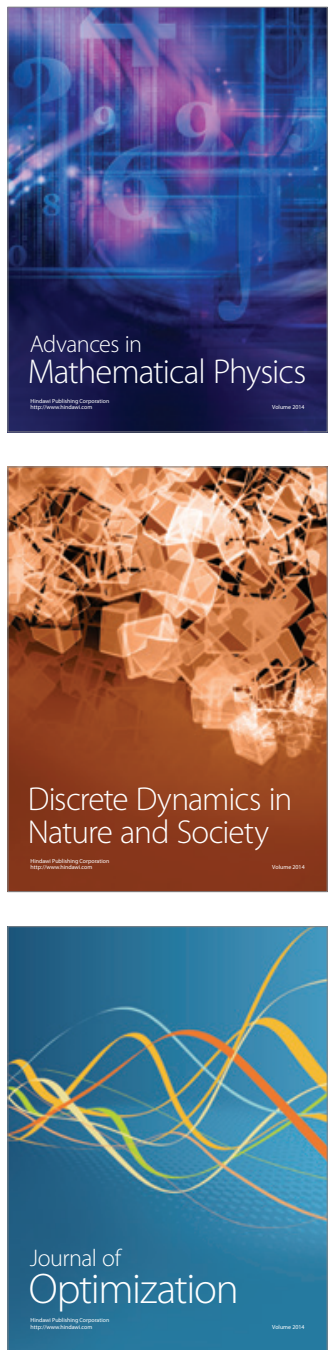